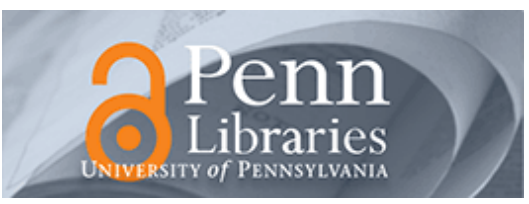

University of Pennsylvania

ScholarlyCommons

June 2004

\title{
Calibrating an Air-Ground Control System from Motion Correspondences
}

\author{
Rahul Rao \\ University of Pennsylvania, rahulrao@grasp.cis.upenn.edu \\ Camillo J. Taylor \\ University of Pennsylvania, cjtaylor@cis.upenn.edu \\ R. Vijay Kumar \\ University of Pennsylvania, kumar@grasp.upenn.edu
}

Follow this and additional works at: https://repository.upenn.edu/cis_papers

\section{Recommended Citation \\ Rahul Rao, Camillo J. Taylor, and R. Vijay Kumar, "Calibrating an Air-Ground Control System from Motion Correspondences", . June 2004.}

Copyright 2004 IEEE. Reprinted from Proceedings of the 2004 IEEE Computer Society Conference on Computer Vision and Pattern Recognition(CVPR 2004), Volume 2, pages 218-225.

Publisher URL: http://ieeexplore.ieee.org/x pl/tocresult.jsp?isNumber=29134\&page=2

This material is posted here with permission of the IEEE. Such permission of the IEEE does not in any way imply IEEE endorsement of any of the University of Pennsylvania's products or services. Internal or personal use of this material is permitted. However, permission to reprint/republish this material for advertising or promotional purposes or for creating new collective works for resale or redistribution must be obtained from the IEEE by writing to pubs-permissions@ieee.org. By choosing to view this document, you agree to all provisions of the copyright laws protecting it.

This paper is posted at ScholarlyCommons. https://repository.upenn.edu/cis_papers/30

For more information, please contact repository@pobox.upenn.edu. 


\title{
Calibrating an Air-Ground Control System from Motion Correspondences
}

\author{
Abstract \\ In this paper we consider the problem of controlling the motion of a vehicle moving on a ground plane \\ based on aerial imagery. In the course of this work we propose a novel analysis of the relationship \\ between the velocity of the vehicle on the ground plane and the velocity of its projection in the image. We \\ show that this relationship provides information about a subset of the parameters of the homography \\ relating the ground plane to the aerial image plane and describe how we can recover this relationship \\ from available measurements.

\section{Comments} \\ Copyright 2004 IEEE. Reprinted from Proceedings of the 2004 IEEE Computer Society Conference on \\ Computer Vision and Pattern Recognition(CVPR 2004), Volume 2, pages 218-225. \\ Publisher URL: http://ieeexplore.ieee.org/x pl/tocresult.jsp?isNumber=29134\&page=2 \\ This material is posted here with permission of the IEEE. Such permission of the IEEE does not in any way \\ imply IEEE endorsement of any of the University of Pennsylvania's products or services. Internal or \\ personal use of this material is permitted. However, permission to reprint/republish this material for \\ advertising or promotional purposes or for creating new collective works for resale or redistribution must \\ be obtained from the IEEE by writing to pubs-permissions@ieee.org. By choosing to view this document, \\ you agree to all provisions of the copyright laws protecting it.
}




\title{
Calibrating an Air-Ground Control System from Motion Correspondences
}

\author{
Rahul Rao Camillo J. Taylor Vijay Kumar \\ General Robotics and Active Sensory Perception (GRASP) Laboratory \\ University of Pennsylvania, Levine Hall, Philadelphia, PA 19104-6228 \\ E-mail: \{rahulrao, cjtaylor, kumar\}@grasp.cis.upenn.edu
}

\begin{abstract}
In this paper we consider the problem of controlling the motion of a vehicle moving on a ground plane based on aerial imagery. In the course of this work we propose a novel analysis of the relationship between the velocity of the vehicle on the ground plane and the velocity of its projection in the image. We show that this relationship provides information about a subset of the parameters of the homography relating the ground plane to the aerial image plane and describe how we can recover this relationship from available measurements.
\end{abstract}

\section{Introduction}

In this paper we consider the problem of controlling the motion of a robot moving on a plane based on the imagery acquired by an overhead camera. The results presented here could, for example, be used to control the motion of unmanned ground vehicles based on imagery acquired by unmanned air vehicles. One of the principal advantages of using aerial imagery for this task is that it typically provides a convenient overall context for defining mission objectives. For example, one can easily select targets to be visited or sketch desired trajectories through the environment in the overhead image.

In order to relate objectives defined in the image plane to actual motion commands provided to the robot, we must be able to relate the velocity commands relayed to the robot to the desired motions in the image plane. One of the contributions of this paper is a novel analysis of the relationship between the velocity of the vehicle on the ground plane and the velocity of its projection in the image plane. Significantly, the analysis provides a metric relationship between these quantities as opposed to a projective mapping. This is particularly useful in the context of control applications where we wish to command or regulate the speed of the robot.

Based on this analysis, we propose an algorithm that can be used to recover the parameters of this relationship from available measurements. Once this relationship has been characterized, it can be used to regulate the robot to a particular target or along a given trajectory. We also explain how

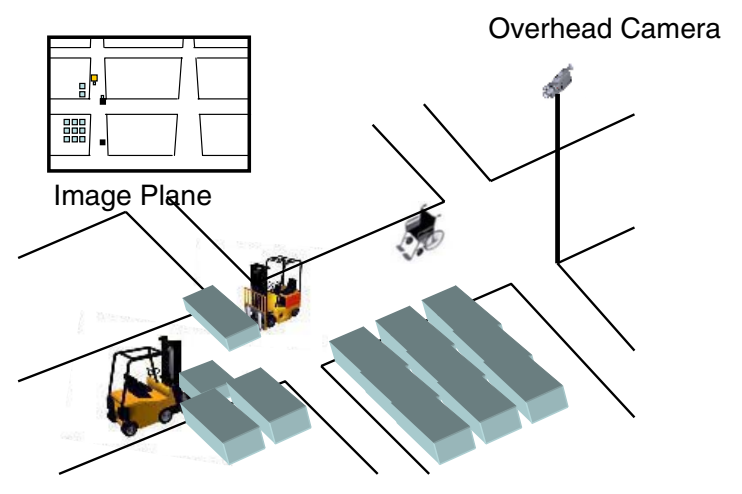

Figure 1: Control of Vehicles in a Warehouse Using an Overhead Camera

\subsection{Related Work}

Vision based control is a well studied topic in the Computer Vision and Robotics literatures. Researchers in this area have proposed a number of schemes for controlling the motion of one or more manipulators based on measurements derived from imaging systems. All of these schemes either directly or indirectly address the problem of characterizing the relationship between the robot's motion and the observed image motion.

Espiau et al and Papanikolopolous describe schemes for approximating this relationship through the image Jacobian or Interaction Screw and show how this matrix can be updated as the robot moves.

Other researchers $[6,8,9,2,10,11,14,4]$ have proposed robust feedback control schemes that are capable of accurately regulating a robotic system to a desired configuration even when the parameters relating the robot frame to the image measurements are only known approximately [7]. 
In this work we consider an important special case of the visual servoing problem where the motion of the robot is confined to a plane. This restriction allows us to precisely characterize the relationship between robot and image velocities globally and to recover the parameters of this relationship online.

Overhead imagery has, of course, been used before to control robot motion. A popular example is the annual table top robotic soccer competition where information from an overhead camera is used to estimate the position and orientation of a group of robots on the playing surface [3]. Since this situation is tightly prescribed, one can make assertions about the relationship between image coordinates and world coordinates a priori.

Dixon et al [5] considered the problem of servoing ground robots based on overhead imagery acquired with a weakly calibrated camera system. However, they restrict their consideration to situations where the image plane is parallel to the ground plane of the robots. This reduces the problem to one of finding a similarity transformation between the image plane and the ground plane (4 parameters) as opposed to the more general projective transformation ( 8 parameters).

Our work improves upon existing techniques by providing an approach that can be applied to the general case of the problem where no assumptions are made about the intrinsic parameters of the camera or the orientation of the image plane with respect to the ground.

Avidan and Shashua [1] tackle the problem of recovering the trajectory of a moving object from point correspondences while Shashua and Wolf [13] describe how the homography tensor relating three frames can be recovered from motion correspondences. Our work differs from these efforts in that we seek to recover a metric relationship between the ground plane velocity of the robot and the corresponding motion in a single aerial image plane.

\section{Analysis}

In this section we investigate the relationship between a vehicles velocity on the ground plane and the velocity of its projection in a fixed overhead camera.

In the sequel, we will assume some means for measuring the velocity of the ground vehicle with respect to a fixed frame. For example, an unmanned ground vehicle equipped with a compass and an odometry system would be able to measure it's heading and speed with respect to a fixed magnetic reference frame. We expect, however, that these velocity measurements will contain errors which make it impractical to deduce the displacement of the robot by integrating velocity readings over time.

A typical situation is represented in Figure 1 where an overhead camera has sight of a ground robot and the desired trajectory. Let $\mathbf{w} \equiv(x, y, 1)^{T}$ denote the homogeneous coordinates of a point on the ground plane and $\mathbf{c}=(u, v, 1)^{T}$ denote the coordinates of the projection of $w$ in the image. It is easy to show that $\mathbf{w}$ and $\mathbf{c}$ are related by a projective transformation $\mathbf{G}$. This can be expressed as

$$
\begin{aligned}
\mathbf{c} & \propto \mathbf{G w}, \mathbf{G} \in G L(3) \\
\Rightarrow \mathbf{w} & \propto \mathbf{H c}
\end{aligned}
$$

where $\mathbf{H}=\mathbf{G}^{-\mathbf{1}}$.

For clarity, the matrices $\mathbf{G}$ and $\mathbf{H}$ be represented in terms of their columns as $\mathbf{G}=\left(\begin{array}{lll}G^{1} & G^{2} & G^{3}\end{array}\right)$ and $\mathbf{H}=\left(\begin{array}{lll}H^{1} & H^{2} & H^{3}\end{array}\right)$ respectively. Similarly, let the rows of $\mathbf{G}$ and $\mathbf{H}$ be represented by $\left(\begin{array}{llll}G_{1} & G_{2} & G_{3}\end{array}\right)$ and $\left(\begin{array}{lll}H_{1} & H_{2} & H_{3}\end{array}\right)$ respectively. Note that superscripts and subscripts are used to distinguish between matrix columns and rows. Replacing the proportionality sign in (2) by an equality we get

$$
\mathbf{w}=\lambda \mathbf{H} \mathbf{c}
$$

where $\lambda=\frac{1}{H_{3} \cdot \mathbf{c}}$. Similarly, the image coordinates, $\left(\begin{array}{ll}u & v\end{array}\right)$, can be expressed in terms of the homogeneous ground plane coordinates, $\mathbf{w}$, as follows:

$$
\begin{aligned}
& u=\frac{G_{1} \cdot \mathbf{w}}{G_{3} \cdot \mathbf{w}} \\
& v=\frac{G_{2} \cdot \mathbf{w}}{G_{3} \cdot \mathbf{w}}
\end{aligned}
$$

Differentiating (4) with respect to time yields

$$
\dot{u}=\frac{\left(G_{3} \cdot \mathbf{w}\right)\left(G_{1} \cdot \dot{\mathbf{w}}\right)-\left(G_{1} \cdot \mathbf{w}\right)\left(G_{3} \cdot \dot{\mathbf{w}}\right)}{\left(G_{3} \cdot \mathbf{w}\right)^{2}} .
$$

Using the expression for $\mathbf{w}$ from (3) we get

$$
\dot{u}=\frac{\left(G_{3} \cdot(\lambda \mathbf{H} \mathbf{c})\right)\left(G_{1} \cdot \dot{\mathbf{w}}\right)-\left(G_{1} \cdot(\lambda \mathbf{H} \mathbf{c})\right)\left(G_{3} \cdot \dot{\mathbf{w}}\right)}{\left(G_{3} \cdot(\lambda \mathbf{H} \mathbf{c})\right)^{2}} .
$$

Note that by definition the matrices $\mathbf{G}$ and $\mathbf{H}$ are inversely related. That is

$$
\begin{aligned}
G \cdot H & =I \\
\Rightarrow\left(\begin{array}{l}
G_{1} \\
G_{2} \\
G_{3}
\end{array}\right) \cdot H & =\left(\begin{array}{l}
G_{1} \cdot H \\
G_{2} \cdot H \\
G_{3} \cdot H
\end{array}\right)=\left(\begin{array}{lll}
1 & 0 & 0 \\
0 & 1 & 0 \\
0 & 0 & 1
\end{array}\right)
\end{aligned}
$$

This implies that $G_{1} \cdot(\mathbf{H} c)=\left(\begin{array}{lll}1 & 0 & 0\end{array}\right) \cdot c=u$ and $G_{3} \cdot(\mathbf{H} c)=\left(\begin{array}{lll}0 & 0 & 1\end{array}\right) \cdot c=1$. Using these facts, and the expression for $\lambda$, we can simplify (7) to yield

$$
\dot{u}=\left(H_{3} \cdot \mathbf{c}\right)\left[G_{1} \cdot \dot{\mathbf{w}}-u\left(G_{3} \cdot \dot{\mathbf{w}}\right)\right]
$$

Similarly

$$
\dot{v}=\left(H_{3} \cdot \mathbf{c}\right)\left[G_{2} \cdot \dot{\mathbf{w}}-v\left(G_{3} \cdot \dot{\mathbf{w}}\right)\right]
$$


These expressions can be written compactly as

$$
\begin{aligned}
\left(\begin{array}{l}
\dot{u} \\
\dot{v}
\end{array}\right) & =\left(H_{3} \cdot \mathbf{c}\right)\left(\begin{array}{l}
G_{1} \cdot \dot{\mathbf{w}}-u\left(G_{3} \cdot \dot{\mathbf{w}}\right) \\
G_{2} \cdot \dot{\mathbf{w}}-v\left(G_{3} \cdot \dot{\mathbf{w}}\right)
\end{array}\right) \\
& =\left(H_{3} \cdot \mathbf{c}\right)\left(\begin{array}{lll}
1 & 0 & -u \\
0 & 1 & -v
\end{array}\right) \mathbf{G} \dot{\mathbf{w}}
\end{aligned}
$$

where $\dot{\mathbf{w}}=(\dot{x}, \dot{y}, 0)^{T}$. This can be rewritten as

$$
\left(\begin{array}{c}
\dot{u} \\
\dot{v}
\end{array}\right)=\left(H_{3} \cdot \mathbf{c}\right)\left(\begin{array}{ccc}
1 & 0 & -u \\
0 & 1 & -v
\end{array}\right)\left(\begin{array}{ll}
G^{1} & G^{2}
\end{array}\right)\left(\begin{array}{c}
\dot{x} \\
\dot{y}
\end{array}\right)
$$

Note that $H_{3}$, the third row of $\mathbf{H}$, can be expressed in terms of the columns of $\mathbf{G}$ as

$$
H_{3}=\frac{G^{1} \times G^{2}}{\left(G^{3}\right) \cdot\left(G^{1} \times G^{2}\right)}=\frac{G^{1} \times G^{2}}{\operatorname{det}(\mathbf{G})}
$$

Since the matrix $\mathbf{G}$ represents a projective transformation, it's scale is immaterial which means that we can, without loss of generality, restrict $\mathbf{G}$ to be a matrix with unit determinant. Alternatively, one can note that scaling the matrix $\mathbf{G}$ by a constant does not affect equation (13). With this restriction, equation (13) becomes:

$$
\left(\begin{array}{c}
\dot{u} \\
\dot{v}
\end{array}\right)=\left(\left(G^{1} \times G^{2}\right) \cdot \mathbf{c}\right)\left(\begin{array}{ccc}
1 & 0 & -u \\
0 & 1 & -v
\end{array}\right)\left(\begin{array}{ll}
G^{1} & G^{2}
\end{array}\right)\left(\begin{array}{l}
\dot{x} \\
\dot{y}
\end{array}\right)
$$

This equation has a number of notable features. It provides a metric relationship between the velocity of the robot on the ground plane $(\dot{x}, \dot{y})$ and the velocity of its projection in the image $(\dot{u}, \dot{v})$. Strangely, it does this without requiring a normalizing division such as the ones implied by the projective relationships given in Equations (1) and (2). The expression reveals that the observed image velocity depends linearly on the ground plane velocity, $(\dot{x}, \dot{y})$, and quadratically on the vectors $G^{1}, G^{2}$ and c.

Note that this expression only involves the first two columns of $\mathbf{G}$ which means that we cannot recover information about the third column of $\mathbf{G}$ solely from measurements of vehicle and image velocities. We can only hope to recover information about 6 of the 8 degrees of freedom that define the homography $\mathbf{G}$. The missing two degrees of freedom can be accounted for by noting that the origin of the ground planes reference frame can be chosen arbitrarily.

\subsection{Calibration from Motion Correspon- dences}

We can recover the vectors $G^{1}$ and $G^{2}$ from image measurements by observing that the right hand side of equation (13) must be perpendicular to the vector $\left.\left(\begin{array}{ll}-\dot{v} & \dot{u}\end{array}\right)\right)$. That is:

$$
\left(\begin{array}{ll}
-\dot{v} & \dot{u}
\end{array}\right) \cdot\left(\begin{array}{ccc}
1 & 0 & -u \\
0 & 1 & -v
\end{array}\right)\left(\begin{array}{ll}
G^{1} & G^{2}
\end{array}\right)\left(\begin{array}{l}
\dot{x} \\
\dot{y}
\end{array}\right)=0
$$

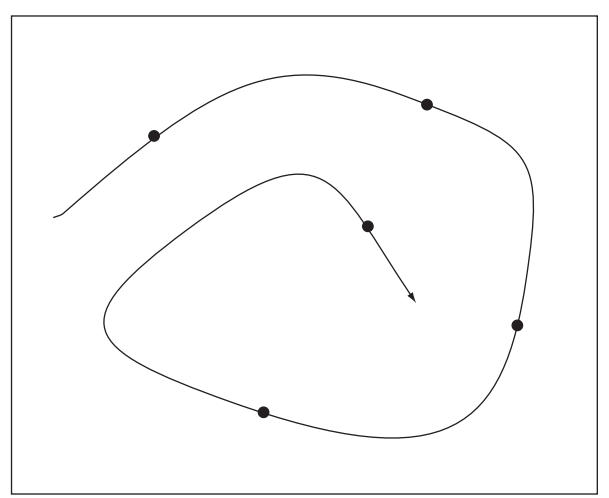

Figure 2: The relationship between the velocity of the vehicle in the plane and the velocity of its projection in the image can be completely determined given a set of five correspondences between the vehicles velocity, $(\dot{x}, \dot{y})$, and its position, $(u, v)$, and motion, $(\dot{u}, \dot{v})$, in the image. This could be accomplished by drawing 5 samples from the robots trajectory as shown above. Alternatively, if multiple robots are being viewed simultaneously, we can imagine recovering many such correspondences at a time.

This homogeneous equation is linear in the unknown vectors $G^{1}$ and $G^{2}$. Given five separate measurements of a vehicles position in the image $(u, v)$, it's instantaneous image velocity, $(\dot{u}, \dot{v})$, and its ground plane velocity, $(\dot{x}, \dot{y})$ as shown in Figure 2 . We can construct a system of linear homogeneous equations which allows us to recover $G^{1}$ and $G^{2}$ up to an unknown scale factor, that is $\left(\begin{array}{ll}G^{1} & G^{2}\end{array}\right)=\alpha\left(\hat{G}^{1} \hat{G}^{2}\right)$. This scale parameter, $\alpha$, can be resolved by substituting the scaled versions of $G^{1}$ and $G^{2}$ into equation (15) and enforcing equality as follows.

$$
\left(\begin{array}{c}
\dot{u} \\
\dot{v}
\end{array}\right)=\alpha^{3}\left(\left(\hat{G}^{1} \times \hat{G}^{2}\right) \cdot \mathbf{c}\right)\left(\begin{array}{ccc}
1 & 0 & -u \\
0 & 1 & -v
\end{array}\right)\left(\begin{array}{ll}
\hat{G}^{1} & \hat{G}^{2}
\end{array}\right)\left(\begin{array}{c}
\dot{x} \\
\dot{y}
\end{array}\right)
$$

\subsection{An Alternate Derivation}

The homogeneous equation given in (16) can also be derived through an appeal to projective geometry. As the robot moves on the ground plane with velocity $(\dot{x}, \dot{y})$ it's projection in the image will appear to move towards the vanishing point, $\mathbf{v}_{p}$, given by $\mathbf{v}_{p} \propto \mathbf{G} \cdot\left(\begin{array}{lll}\dot{x} & \dot{y} & 0\end{array}\right)^{T}$.

The following expression yields the projective coordinates of the line, 1 , passing through $(u, v)$ in the direction $(\dot{u}, \dot{v})$ :

$$
\mathbf{l} \propto\left(\begin{array}{c}
u \\
v \\
1
\end{array}\right) \times\left(\begin{array}{c}
\dot{u} \\
\dot{v} \\
0
\end{array}\right)
$$


The vanishing point $\mathbf{v}_{p}$ must lie on the line defined by 1. This constraint is expressed succinctly by the following homogeneous equation.

$$
\mathbf{l}^{T} \mathbf{G}\left(\begin{array}{c}
\dot{x} \\
\dot{y} \\
0
\end{array}\right)=0
$$

Further simplifying (19) leads to an expression only in terms of the first two columns of $\mathbf{G}$ rather than the entire homography.

$$
\left(\left(\begin{array}{l}
u \\
v \\
1
\end{array}\right) \times\left(\begin{array}{c}
\dot{u} \\
\dot{v} \\
0
\end{array}\right)\right)^{T}\left(\begin{array}{ll}
G^{1} & G^{2}
\end{array}\right)\left(\begin{array}{l}
\dot{x} \\
\dot{y}
\end{array}\right)^{T}=0
$$

This expression is identical in form to equation (16). However, this line of reasoning does not provide the metric relationship between $(\dot{x}, \dot{y})$ and $(\dot{u}, \dot{v})$ given in equation (15).

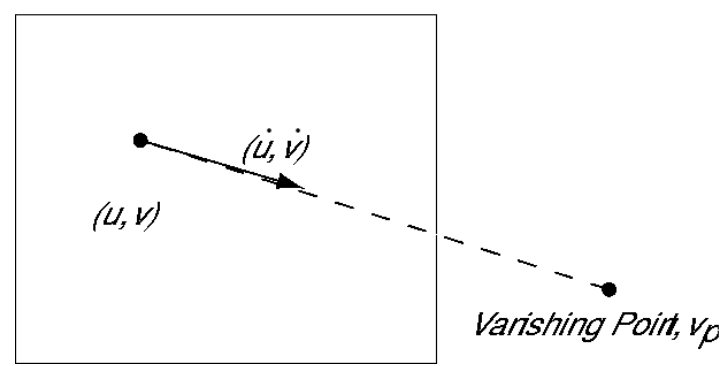

Figure 3: The relationship between the line at infinity on the ground plane and its projection in the image can be recovered from the correspondences between vehicle velocities and image velocities by noting that any time the projection of the vanishing point $\mathbf{v}_{p}$ must lie along the line passing through the current projection of the robot, $(u, v)$, in the direction $(\dot{u}, \dot{v})$.

\section{Experimental Results}

In order to investigate the efficacy of the proposed technique experiments were performed both in simulation and with an actual robotic platform.

Section 3.1 reports the results of the tests run in simulation, while section 3.2 briefly describes the results that were obtained from experimental tests with an actual robot.

As has been outlined in Section 2.1 the vectors $G^{1}$ and $G^{2}$ can be recovered from at least five distinct measurements of $(u, v, \dot{u}, \dot{v}, \dot{x}, \dot{y})$ as the robot moves on the ground. Our tests gave us an idea of the accuracy of the results returned by our online estimation procedure.

\subsection{Simulation Experiments}

In our simulations, our virtual robot was commanded to move along 8 distinct directions (we will refer to them as segments). Virtual measurements were collected in each segment. The robot's forward velocity $(V)$ was set at 0.12 $\mathrm{m} / \mathrm{s}$. To account for uncertainty in the image pixel data, we introduced random noise of up to 5 pixels in the image in both directions. We also assumed that we did not have precise information about the velocity or the orientation of the robot and introduced additive white noise of up to $10 \%$ of the robot's forward velocity to account for this. These uncertainties were chosen to closely duplicate real experimental conditions.

Although the calibration procedure would work for random motions of the robot, we arranged the segments in the simulation at $30^{\circ}$ increments.

In our simulation experiments we compared the values returned by the estimation procedure to the known ground truth values. We used two benchmarks to compare the results that we obtain from simulation to the ideal results. Let g denote a $3 \times 2$ matrix representing the first two columns of the homography $\mathbf{G}$, appropriately scaled, i.e.

$$
\mathbf{g}=\frac{1}{\sqrt[3]{\operatorname{det}(\mathbf{G})}}\left(G^{1} \quad G^{2}\right)
$$

$\hat{\mathrm{g}}$ denotes the estimates derived from the noisy measurements by our procedure. These 2 values were compared using the following statistic.

$$
n=\frac{\|\mathbf{g}-\hat{\mathbf{g}}\|_{F}}{\|\mathbf{g}\|_{F}}
$$

We ran 100 tests in simulation and measured this value for each test. The mean value, $\bar{n}$, for the 100 tests in simulation, was found to be $\bar{n}=0.2519$. The median value, $\tilde{n}$, was found to be $\tilde{n}=0.2292$. A typical set of values as recovered in simulation is presented in Table 1 alongside the values of the homography as obtained by point correspondences. A histogram representing the computed values of $n$ over the 100 simulations is shown in Figure 4

Table 1: A Sample Comparison of Ideal and Simulated Values of the Limited Homography Parameters

\begin{tabular}{|c|c|c|c|}
\hline$\hat{G}^{1}($ ideal $)$ & $\hat{G}^{1}($ sim $)$ & $\hat{G}^{2}($ ideal $)$ & $\hat{G}^{2}($ sim $)$ \\
\hline-8.8347 & -9.4843 & -2.0486 & -2.5053 \\
\hline-0.2190 & -0.4463 & 3.3972 & 3.5419 \\
\hline-0.00065 & -0.0022 & -0.0048 & -0.0052 \\
\hline
\end{tabular}

Next, we computed image velocities as represented in equation (17) for each of these simulation tests in the presence of noise (call them $(\dot{\hat{u}}, \dot{\hat{v}}))$. These values were then 


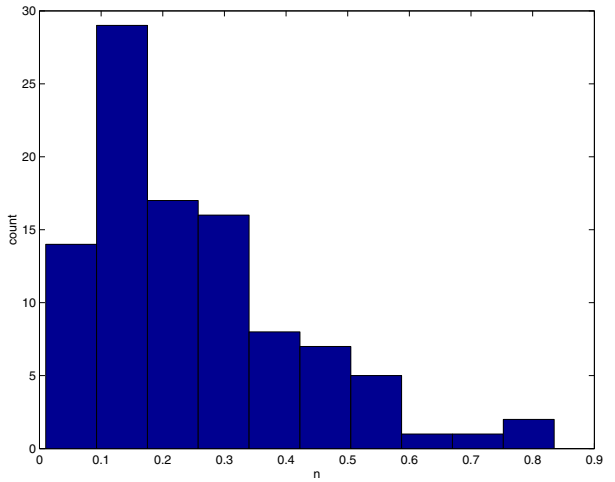

Figure 4: Distribution of the error metric computed in the simulations, $n=\frac{\|\mathbf{g}-\hat{\mathbf{g}}\|_{F}}{\|\mathbf{g}\|_{F}}$

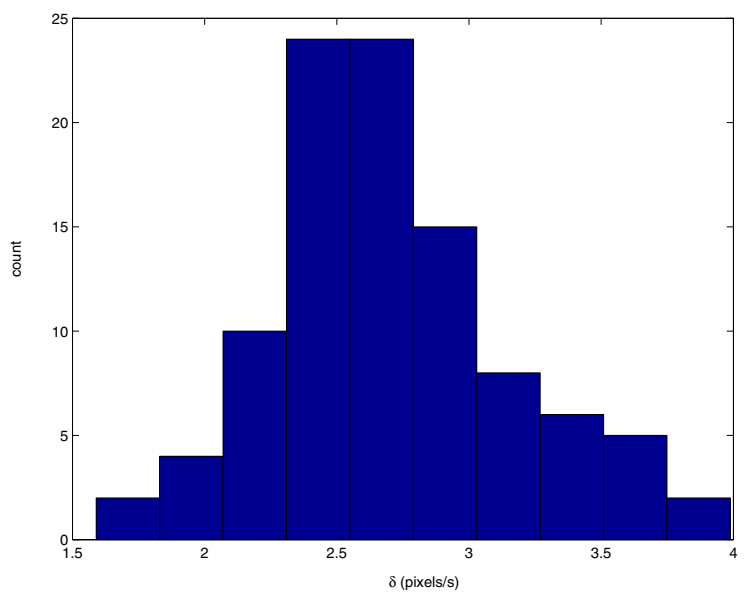

Figure 5: Distribution of the image velocity error norm, $\delta$, computed in the simulations.

compared with ideal values, i.e. values obtained in the absence of all noise, $(\dot{u}, \dot{v})$. The discrepancies were then computed and a norm metric was computed as

$$
\delta=\|(\dot{u}-\dot{\hat{u}}),(\dot{v}-\dot{\hat{v}})\|
$$

for each of the 100 tests. The mean and median values of $\delta$ were determined to be 2.7016 pixels/s and 2.6592 pixels/s over all the tests.

To give a better idea of the discrepancy between the values recovered from simulation and the ideal values, we present a histogram in Figure 5 that shows the distribution of the calculated error norm, $\delta$.

We now present similar results from experiments that were conducted with the robot moving on the ground, capturing the required data in each of the segments.

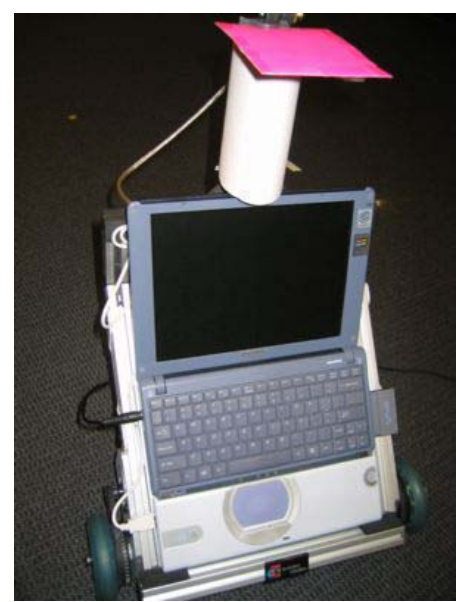

Figure 6: The ER1 robot used for experimental runs.

\subsection{Mobile Robot Experiments}

We conducted a series of experiments with the ER-1 mobile robot from Evolution Robotics and an overhead Dragonfly firewire camera with a Sony ICX204 sensor. The ER-1 is a three- wheeled, Hilare-like robot and is shown in Figure 6.

As mentioned in Section 3.1, the robot was moved in increments of $30^{\circ}$ for 8 segments. The path of the robot along with a camera view of the robot as it moves during calibration are shown in Figure 7. It was continuously tracked, thus obtaining image information as well as real time ground velocity information. The parameters of the limited homography were then computed as outlined in Section 2.1. We conducted 20 experiments with the actual robot and recovered the values of the homography for each case.

We compared the results that were obtained from our online calibration procedure, $\hat{\mathrm{g}}$, with an estimate for the ground plane homography computed from point correspondences, $\tilde{\mathbf{G}}$. We used the same benchmark described in section 3.1 to judge the accuracy of the recovered parameters. Specifically, we computed the quantity,

$$
n_{e}=\frac{\|\tilde{\mathbf{g}}-\hat{\mathbf{g}}\|_{F}}{\|\tilde{\mathbf{g}}\|_{F}}
$$

Over the 20 experiments tests that were conducted, the mean and median values of $n_{e}$ were determined to be 0.0563 and 0.0540 respectively.

A typical set of values for the recovered homography parameters as compared with the ideal parameters are presented in Table 2. 

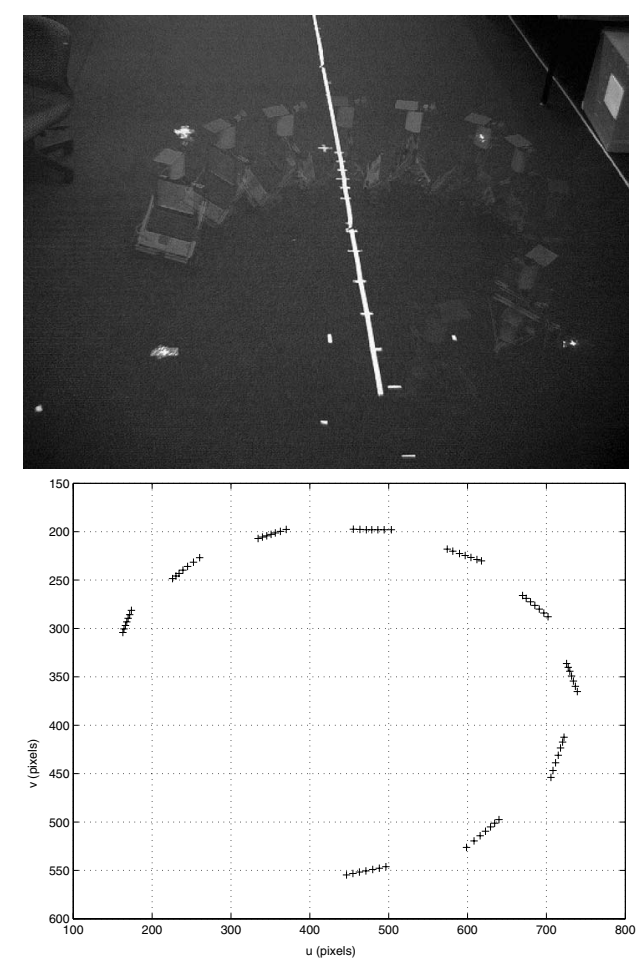

Figure 7: The robot as seen by the overhead camera (top) and the path traced by it (bottom) during a calibration run.

Table 2: A Sample Comparison of Ideal and Experimental Values of the Limited Homography

\begin{tabular}{|c|c|c|c|}
\hline$\hat{G}^{1}($ ideal $)$ & $\hat{G}^{1}(\exp )$ & $\hat{G}^{2}($ ideal $)$ & $\hat{G}^{2}(\exp )$ \\
\hline-8.8347 & -8.9873 & -2.0486 & -2.1911 \\
\hline-0.2190 & -0.4488 & 3.3972 & 3.3299 \\
\hline-0.00064 & -0.0008 & -0.0048 & -0.0049 \\
\hline
\end{tabular}

\section{Applications}

The previous analysis showed that the relationship between the velocity of the vehicle in the world and its motion in the image is completely determined by the two vectors $G^{1}$ and $G^{2}$. Furthermore it shows how these parameters can be recovered from the available measurements in a real world setting.

Once the parameters $G^{1}$ and $G^{2}$ have been recovered, they can be used to relate velocities in the image to velocities on the ground plane and vice versa. From the standpoint of control applications this is a very useful capability. It allows us to define and execute regulation strategies in the image plane since we can relate desired image velocities to the velocity commands required to achieve them. This means that we can guide the robot to approach targets or follow trajectories defined in the image plane. We can also use this relationship to regulate the velocity of other vehicles moving on the ground plane since we can deduce the vehicles ground plane velocity from its image velocity.

\subsection{Simulation Results}

A series of simulation experiments were carried out to test online control schemes based on the ideas presented in this work. In each of the experiments a non-holonomic unicycle robot was guided along a trajectory defined in the image using the estimates for $G^{1}$ and $G^{2}$ recovered in a previous stage. The starting pose of the robot is chosen arbitrarily within the camera's field of view.

The design of the controller used in these experiments uses ideas from nonlinear control theory and differential flatness to transform the control problem from the real world to the image space and then generate a controller in the image space [12].

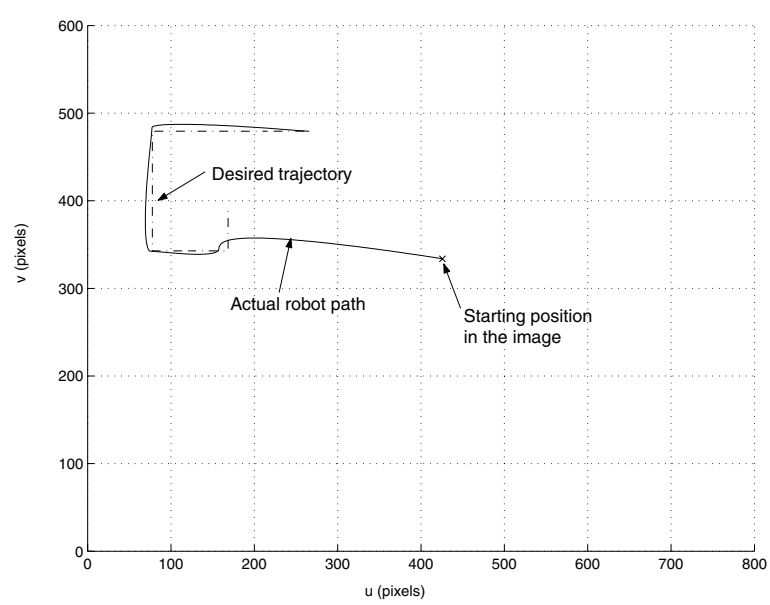

Figure 8: The robot path as seen in the image (solid) and the desired image plane trajectory

Figure 8 shows the path traced by the robot, as seen in the image, under the influence of the controller. The starting position of the robot is indicated by a cross-hair and it can be seen that the robot does not start on the desired trajectory but converges toward it.

Another example is shown in Figure 9. In this case too, the robot's initial position and orientation in the real world are indicated by the line and the cross- hairs. The forward and angular velocities of the robot over time are shown in Figure 10.

\subsection{Controlling the ER1 Robot}

Using the calibration routine described in Section 2.1 we were able to experimentally obtain a partial calibration of 


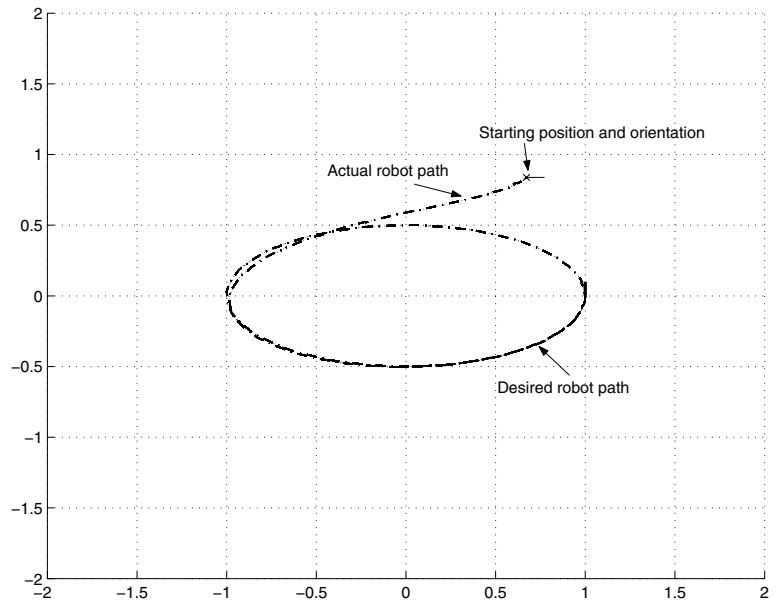

Figure 9: The robot path (dot-dash) and the desired elliptical trajectory in the real world

the camera and then use these calibration parameters to control the motion of a robot from a starting point to a desired point. The robot's destination was specified in the image and the feedback controller relied on continuous image feedback regarding the robot's position to guide it to it's desired goal. Once the goal was specified, a simple image based feedback linearizing controller utilizing the recovered homography parameters enabled the robot to reach its goal.

The point-to-point controller was tested on the experimental platform. A typical path followed by the robot in reaching several intermediate goals is presented in Figure 11. The dotted lines in the figure indicate the path traced by the robot during the calibration routine (note that the turns are now random) while the solid lines indicate the path taken by the robot from one point to another.

\subsection{Extension to a Moving Observer}

In the preceding analysis we assumed that the overhead camera remained stationary during the motion of the ground vehicle. This restriction can be removed if we posit the existence of a set of 4 or more fixed points on the ground plane which can be identified and tracked over time. These point correspondences can be used to compute collineations that effectively fixate the ground plane in the image, a simple form of image stabilization. Note that we do not require any information about the location of the tracked points on the ground plane since they are only being used to compute collineations between images. This stabilization procedure returns us to the realm where the stationary camera analysis can be applied.
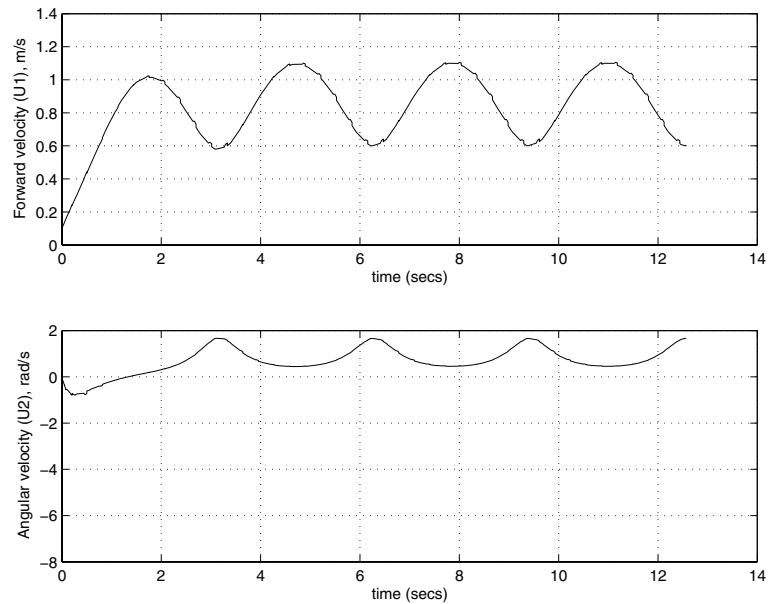

Figure 10: The forward and angular velocities of the robot while tracking the desired trajectory in Fig. 9

\section{Conclusions}

This paper considers the problem of controlling the motion of a vehicle moving on a ground plane based on the imagery acquired with an overhead camera, an important special case of the visual servoing problem. One of the primary motivations for considering this situation is the fact that overhead imagery provides a convenient context for defining motion objectives for the robot. In order to relate objectives defined in the image plane to the velocity commands passed to the vehicle we must first recover the relationship between the vehicles velocity and the motion of its projection in the image. To this end, we propose an novel analysis of this relationship which yields some interesting insights. The analysis shows that the image velocity depends linearly upon its ground plane velocity, $(\dot{x}, \dot{y})$, and quadratically upon the location of its projection in the image, $(u, v)$, and a subset of the parameters of the homography relating the ground and image planes, $\left(G^{1}, G^{2}\right)$.

We describe how the relationship can be calibrated from available measurements. Once this relationship has been recovered, it can be exploited to carry out a variety of image guided control tasks. The approach can also be extended to handle the case where the overhead camera moves over time by performing an image based stabilization procedure to fixate the ground plane in the image sequence.

Our experimental results both in simulation and on an actual robot show that the scheme performs well even in the presence of measurement errors and can be used for practical control tasks. 


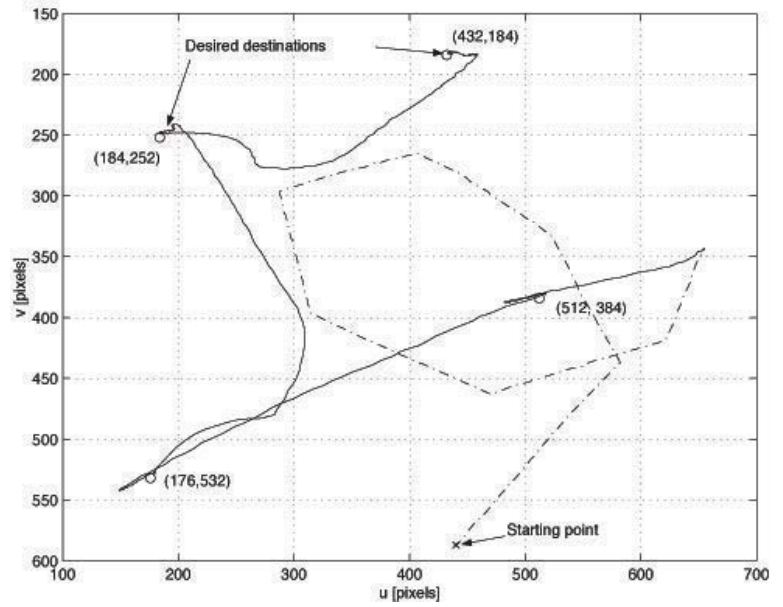

Figure 11: The robot's path during calibration (dotted lines) and during point to point navigation (solid lines). Intermediate destinations chosen at random by the user are indicated by circles.

\section{References}

[1] S. Avidan and A. Shashua. Trajectory triangulation: Reconstruction of moving points from a monocular image sequence. IEEE Trans. Pattern Anal. Machine Intell., 22(4):348-357, 2000.

[2] Ronen Basri, E. Rivlin, and I. Shimshoni. Visual homing: surfing on the epipoles. In International Conference on Computer Vision, 1998.

[3] R. D'Andrea. Robot soccer: A platform for systems engineering. Computers in Education Journal, 10(1):57-61, 2000.

[4] Koichiro Deguchi. Optimal motion control for image-based visual servoing by decoupling translation and rotation. In Proceedings of the 1998 IEEE/RSJ International Conference on Intelligent Robots and Systems, page 705, 1998.

[5] W.E. Dixon, D.M. Dawson, E. Zergeroglu, and A. Behal. Adaptive tracking control of a wheeled mobile robot via an uncalibrated camera system. SMC-B, 31(3):341-352, June 2001.

[6] G. Hager, W. Chang, and A.S. Morse. Robot feedback control based on stereo vision: Towards calibration-free hand-eye coordination. IEEE Control Systems Magazine, 15(1):30-39, 1995

[7] Gregory D. Hager. Calibration-free visual control using projective invariance. In Proc. IEEE Conf. on Comp. Vision and Patt. Recog., pages 1009-1015, 1995.

[8] Nicholas Hollinghurst and Roberto Cipolla. Uncalibrated stereo hand-eye coordination. Image and Vision Computing, 12(3):187-192, April 1994.

[9] R. Horaud, F. Dornaika, and B. Espiau. Visually guided object grasping. IEEE Trans. on Robotics and Automation, 14(4):525-533, August 1998.

[10] Ezio Malis, Francois Chaumette, and Sylvie Boudet. 2d 1/2 visual servoing stability analysis with respect to camera calibration errors. In Proceedings of the 1998 IEEE/RSJ International Conference on Intelligent Robots and Systems, page 691, 1998.
[11] Ezio Malis, Francois Chaumette, and Sylvie Boudet. Positioning of a coarse-calibrated camera with respect to an unkown object by $2 \mathrm{~d} 1 / 2$ visual servoing. In IEEE Int. Conf. on Robotics and Automation, page 1352, 1998.

[12] Rahul Rao, Vijay Kumar, and Camillo Taylor. Visual servoing of a UGV from a UAV using differential flatness. In IEEE Int. Workshop on Intelligent Robots and Systems. IEEE, 2003.

[13] Amnon Shashua and Lior Wolf. Homography tensors: On algebraic entities that represent three views of static or moving planar points. In European Conference on Computer Vision, June 2000.

[14] S. Soatto and P. Perona. Structure-independent visual motion control on the essential manifold. In Proc. of the IFAC Symposium on Robot Control (SYROCO), Capri, Italy, pages 869-876, Sept 1994. 